\title{
Research Article \\ Solving a Nonlinear Fredholm Integral Equation via an Orthogonal Metric
}

\author{
Arul Joseph Gnanaprakasam $\left(\mathbb{D},{ }^{1}\right.$ Gunaseelan Mani $\mathbb{D},^{2}$ Vahid Parvaneh $\mathbb{D},^{3}$ \\ and Hassen Aydi iD $\mathbf{4 , 5 , 6}$ \\ ${ }^{1}$ Department of Mathematics, College of Engineering and Technology, Faculty of Engineering and Technology, SRM Institute of \\ Science and Technology, SRM Nagar, Kattankulathur 603203, Kanchipuram, Chennai, Tamil Nadu, India \\ ${ }^{2}$ Department of Mathematics, Sri Sankara Arts and Science College (Autonomous), Affiliated to Madras University, Enathur, \\ 631 561, Kanchipuram, Tamil Nadu, India \\ ${ }^{3}$ Department of Mathematics, Gilan-E-Gharb Branch, Islamic Azad University, Gilan-E-Gharb, Iran \\ ${ }^{4}$ Université de Sousse, Institut Supérieur d'Informatique et Des Techniques de Communication, H. Sousse 4000, Tunisia \\ ${ }^{5}$ China Medical University Hospital, China Medical University, Taichung 40402, Taiwan \\ ${ }^{6}$ Department of Mathematics and Applied Mathematics, Sefako Makgatho Health Sciences University, Ga-Rankuwa, South Africa
}

Correspondence should be addressed to Vahid Parvaneh; zam.dalahoo@gmail.com and Hassen Aydi; hassen.aydi@isima.rnu.tn

Received 14 June 2021; Accepted 26 August 2021; Published 11 September 2021

Academic Editor: Sergey Shmarev

Copyright (c) 2021 Arul Joseph Gnanaprakasam et al. This is an open access article distributed under the Creative Commons Attribution License, which permits unrestricted use, distribution, and reproduction in any medium, provided the original work is properly cited.

In this paper, we prove fixed point theorems using orthogonal triangular $\alpha$-admissibility on orthogonal complete metric spaces. Some of the well-known outcomes in the literature are generalized and expanded by the obtained results. An instance to help our outcome is being presented.

\section{Introduction}

One of the most important results of mathematical analysis is the famous fixed point result, called the Banach contraction principle (BCP). In several branches of mathematics, it is the most commonly used fixed point result, and it is generalized in many different directions. The substitution of the metric space by other generalized metric spaces is one natural way of reinforcing the $\mathrm{BCP}$. As a generalization of the $\mathrm{BCP}$, Wardowski [1] gave a fixed point result in the setting of complete metric spaces. In other branches of mathematics, the notion of an orthogonal set has many applications and has several kinds of orthogonality. Gordji et al. [2] have imported the current concept of orthogonality on metric spaces and established some fixed point results equipped with the new orthogonality. Furthermore, they used these results to ensure the presence and uniqueness of the solution of a first-ordinary differential equation, while the BCP cannot be applied to this problem. In generalized orthogonal metric spaces, Eshaghi Gordji and Habibi [3] continued in this direction and gave further fixed point theorems. The new definition of orthogonal $F$-contraction mappings was introduced by Sawangsup et al. [4], and some related fixed point theorems on orthogonal-complete metric spaces have been proved. Many authors have investigated orthogonal contractive form mappings, and significant results have been obtained. For more details, see the works of Eshaghi and Habibi [5], Gungor and Turkoglu [6], Yamaod and Sintunavarat [7], Javed et al. [8], Sawangsup and Sintunavarat [9], Senapati et al. [10], Gunaseelan et al. [11], Beg et al. [12], Uddin et al. [13], Ali et al. [14], etc. In this paper, we prove fixed point theorems using orthogonally triangular $\alpha$-admissibility on orthogonal metric spaces. At the end, an application is presented. 


\section{Preliminaries}

The goal of this section is to immediate some concepts and results used in the article. In this article, $\mho, \mathbb{R}^{+}$, and $\mathbb{N}$ denote, respectively, the nonempty set, the positive real numbers, and the set of positive integers.

In 2013, Hussain et al. [15] introduced the concepts of $\alpha$ -admissible mappings and proved some fixed point theorems.

On the other hand, the definition of an orthogonal set (or, $O$-set), some examples, and some premises of orthogonal sets were introduced by Gordji et al. [2], as follows.

Definition 1 (see [2]). Let $\mho \neq \phi$ and $\perp \subseteq \mho \times \mho$ be a binary relation. If $\perp$ satisfies the consecutive condition:

$$
\exists \Re_{0} \in \mho:\left(\forall \Re \in \mho, \Re \perp \Re_{0}\right) \text { or }\left(\forall \Re \in \mho, \Re_{0} \perp \mathfrak{R}\right),
$$

then, it is said to be an orthogonal set (briefly $O$-set). We indicate this $O$-set by $(\mho, \perp)$.

Example 1 (see [2]). Let $\mho=[0, \infty)$ and define $\mathfrak{R} \perp \mathscr{Y}$ if $\Re$ $\mathscr{Y} \in\{\Re, \mathscr{Y}\}$. Then, by setting $\mathfrak{R}_{0}=0$ or $\mathfrak{R}_{0}=1,(\mho, \perp)$ is an $O$-set.

Definition 2 (see [2]). The triplet $(\mho, \perp, \varphi)$ is said to be an $O$ -metric space if $(\mho, \perp)$ is an $O$-set and $(\mho, \varphi)$ is a metric space.

Definition 3 (see [2]). Let $(\mho, \perp, \varphi)$ be an $O$-metric space. Then, if every Cauchy $O$-sequence is convergent, $\mho$ is said to be an orthogonal complete (briefly, $\mathrm{O}$-complete).

Definition 4 (see [2]). Let $(\mho, \perp)$ be an $O$-set. A mapping $\mathfrak{G}: \mho \longrightarrow \mho$ is said to be $\perp$-preserving if $\mathfrak{G} \mathfrak{R} \perp \mathfrak{G} \mathscr{Y}$, whenever $\mathfrak{R} \perp \mathcal{Y}$.

Definition 5 (see [16]). Let $(\mho, \perp)$ be an $O$-set and $\varphi$ be a metric on $\mho, \mathscr{S}: \mho \longrightarrow \mho$ and $\alpha: X \times X \longrightarrow[0, \infty)$ be two mappings. We say that $\mathfrak{G}$ is orthogonally $\alpha$-admissible whenever $\mathfrak{R} \perp \mathscr{Y}$ and $\alpha(\mathfrak{R}, \mathscr{Y}) \geq 1$ imply that $\alpha(\mathfrak{G}(\mathfrak{R}), \mathfrak{G}($ y) $) \geq 1$

Definition 6. Let $(\mho, \perp)$ be an $O$-set and $\varphi$ be a metric on $\mho$. Given $\mathbb{G}: \mho \longrightarrow \mho$ and $\alpha: \mathfrak{R} \times \mathfrak{R} \longrightarrow(-\infty, \infty)$. We say that $\mathfrak{G}$ is an orthogonally triangular $\alpha$-admissible mapping if

(i) $\mathfrak{R} \perp \mathscr{Y}$ and $\alpha(\mathfrak{R}, \mathscr{Y}) \geq 1$ imply that $\alpha(\mathfrak{G}(\mathfrak{R}), \mathfrak{G}(\mathscr{Y}))$ $\geq 1$

(ii) $\mathfrak{R} \perp \mathscr{Z}, \alpha(\Re, \mathscr{Z}) \geq 1$ and $\mathscr{Z} \perp \mathscr{Y}, \alpha(\mathscr{Z}, \mathscr{Y}) \geq 1$ imply that $\mathfrak{R} \perp \mathcal{Y}$

$$
\alpha(\mathfrak{G}(\mathfrak{R}), \mathfrak{G}(\mathscr{Y})) \geq 1 .
$$

\section{Main Results}

Inspired by the $\alpha$-admissibility and fixed point theorems proved by Hussain et al. [15], we prove some fixed point theorems in an orthogonal complete metric space.

Theorem 7. Let $(\mho, \perp, \varphi)$ be an O-complete metric space. Given an orthogonal element $\mathfrak{R}_{0}$. The mappings $\mathfrak{G}: \mho \longrightarrow$ $\mho$ and $\alpha: \mho \times \mho \longrightarrow[0, \infty)$ are such that

$$
\begin{aligned}
& \forall \mathfrak{R}, \mathscr{Y} \in \text { Jwith } \mathfrak{R} \perp \mathcal{Y}, \varphi(\mathfrak{G} \mathfrak{R}, \mathfrak{G} \mathscr{Y}) \\
& \quad>0 \Rightarrow(\varphi(\mathfrak{G} \mathfrak{R}, \mathfrak{G} \mathscr{Y})+\mathfrak{l})^{\alpha(\mathfrak{R}, \mathfrak{G} \mathfrak{R}) \alpha(\mathscr{y}, \mathfrak{G} \mathscr{Y})} \\
& \quad \leq \hbar(\varphi(\mathfrak{R}, \mathscr{Y})) \varphi(\mathfrak{R}, \mathscr{Y})+\mathfrak{l},
\end{aligned}
$$

where $\mathfrak{l} \geq 1$. Suppose there is $\hbar:[0, \infty) \longrightarrow[0,1]$ so that for each bounded positive sequence $\left\{\Omega_{\mathfrak{n}}\right\}, \hbar\left(\Omega_{\mathfrak{n}}\right) \longrightarrow 1$ implies $\Omega_{\mathfrak{n}} \longrightarrow 0$. Suppose that

(1) $G S$ is $\perp$-preserving

(2) $\mathfrak{G}$ is orthogonally triangular $\alpha$-admissible

(3) There exists $\mathfrak{R}_{0} \in \mho$ such that $\mathfrak{R}_{0} \perp \mathfrak{G} \mathfrak{R}_{0}$ and $\alpha\left(\mathfrak{R}_{0}\right.$, $\left(\mathfrak{S} \Re_{0}\right) \geq 1$

(4) Either $\mathfrak{G}$ is orthogonally continuous, or if $\left\{\mathfrak{R}_{\mathfrak{n}}\right\}$ is a sequence in $\mho$ such that $\mathfrak{R}_{\mathfrak{n}} \longrightarrow \mathfrak{R}, \alpha\left(\mathfrak{R}_{\mathfrak{n}}, \mathfrak{R}_{\mathfrak{n}+1}\right) \geq$ 1 for all $\mathfrak{n}$, then $\mathfrak{R}_{\mathfrak{n}} \perp \mathfrak{R}$ and $\alpha(\mathfrak{R},(\mathfrak{S} \Re) \geq 1$

Then, $\mathfrak{G}$ has a fixed point.

Proof. By condition (3), there exists $\mathfrak{R}_{0} \in \mho$ such that $\mathfrak{R}_{0} \perp$ $\mathfrak{G} \mathfrak{R}_{0}$ and $\alpha\left(\mathfrak{R}_{0}, \mathfrak{G} \mathfrak{R}_{0}\right) \geq 1$. Let

$\mathfrak{R}_{1}:=\mathfrak{G} \mathfrak{R}_{0}, \mathfrak{R}_{2}:=\mathfrak{G} \mathfrak{R}_{1}=\mathfrak{G S}^{2} \mathfrak{R}_{0} \ldots \ldots, \mathfrak{R}_{n+1}:=\mathfrak{G} \mathfrak{R}_{n}=\mathfrak{G}^{\mathfrak{n}+1} \mathfrak{R}_{0}$,

for all $\mathfrak{n} \geq 0$. Since $\mathfrak{G S}$ is $\perp$-preserving, then, $\left\{\mathfrak{R}_{n}\right\}$ is an $O$ -sequence in $\mho$. Condition (2) implies that $\alpha\left(\mathfrak{R}_{\mathfrak{n}}, \mathfrak{R}_{\mathfrak{n}+1}\right) \geq$ 1 for all $\mathfrak{n} \geq 0$. If $\mathfrak{R}_{\mathfrak{n}}=\mathfrak{R}_{\mathfrak{n}+1}$ for some $\mathfrak{n} \geq 0$, then, $\boldsymbol{R}_{\mathfrak{n}}$ is a fixed point of $\mathfrak{G}$. Assume that $\mathfrak{R}_{\mathfrak{n}} \neq \mathfrak{R}_{\mathfrak{n}+1}, \forall \mathfrak{n} \geq 0$. Since $\mathfrak{G}$ is $\alpha$-admissible and $\alpha\left(\mathfrak{R}_{0}, \mathfrak{G} \Re_{0}\right) \geq 1$, we deduce that $\alpha\left(\mathfrak{R}_{1}\right.$, $\left.\mathfrak{R}_{2}\right)=\alpha\left(\mathfrak{G} \mathfrak{R}_{0}, \mathfrak{S}^{2} \mathfrak{R}_{0}\right) \geq 1$. By continuing this process, we get $\alpha\left(\mathfrak{R}_{\mathfrak{n}},\left(\mathfrak{S} \mathfrak{R}_{\mathfrak{n}}\right) \geq 1\right.$ for all $\mathfrak{n} \geq 0$. By the inequality (3), we obtain

$$
\begin{aligned}
& \varphi\left(\mathfrak{G} \mathfrak{R}_{\mathfrak{n}-1}, \mathfrak{G} \mathfrak{R}_{\mathfrak{n}}\right)+\mathfrak{l} \leq\left(\varphi\left(\mathfrak{G} \mathfrak{R}_{\mathfrak{n}-1}, \mathfrak{G} \mathfrak{R}_{\mathfrak{n}}\right)+\mathfrak{l}\right)^{\alpha\left(\mathfrak{R}_{\mathfrak{n}-1}, \mathfrak{G} \mathfrak{R}_{\mathfrak{n}-1}\right) \alpha\left(\mathfrak{R}_{\mathfrak{n}}, \mathfrak{G} \mathfrak{R}_{\mathfrak{n}}\right)} \\
& \leq \hbar\left(\varphi\left(\Re_{\mathfrak{n}-1}, \mathfrak{R}_{\mathfrak{n}}\right)\right) \varphi\left(\mathfrak{R}_{\mathfrak{n}-1}, \mathfrak{R}_{\mathfrak{n}}\right)+\mathfrak{l},
\end{aligned}
$$

then

$$
\varphi\left(\Re_{\mathfrak{n}}, \mathfrak{R}_{\mathfrak{n}+1}\right) \leq \hbar\left(\varphi\left(\mathfrak{R}_{\mathfrak{n}-1}, \mathfrak{R}_{\mathfrak{n}}\right)\right) \varphi\left(\mathfrak{R}_{\mathfrak{n}-1}, \mathfrak{R}_{\mathfrak{n}}\right) .
$$

This implies that $\varphi\left(\Re_{\mathfrak{n}}, \mathfrak{R}_{\mathfrak{n}+1}\right) \leq \varphi\left(\boldsymbol{R}_{\mathfrak{n}-1}, \mathfrak{R}_{\mathfrak{n}}\right)$. It follows that the sequence defined by $\left[\varphi\left(\mathfrak{R}_{\mathfrak{n}}, \boldsymbol{R}_{\mathfrak{n}+1}\right)\right]$ is decreasing, hence, there is $\mathfrak{d} \geq 0$ so that $\lim _{\mathfrak{n} \longrightarrow \infty} \varphi\left(\Re_{\mathfrak{n}}, \mathfrak{R}_{\mathfrak{n}+1}\right)=\mathfrak{d}$. We 
claim that $\mathfrak{d}=0$. From (6), we have

$$
\frac{\varphi\left(\mathfrak{R}_{\mathfrak{n}}, \mathfrak{R}_{\mathfrak{n}+1}\right)}{\varphi\left(\mathfrak{R}_{\mathfrak{n}-1}, \mathfrak{R}_{\mathfrak{n}}\right)} \leq \hbar\left(\varphi\left(\boldsymbol{R}_{\mathfrak{n}-1}, \mathfrak{R}_{\mathfrak{n}}\right)\right) \leq 1,
$$

which implies $\lim _{\mathfrak{n} \longrightarrow \infty} \hbar\left(\varphi\left(\mathfrak{R}_{\mathfrak{n}-1}, \mathfrak{R}_{\mathfrak{n}}\right)\right)=1$. We deduce that

$$
\lim _{\mathfrak{n} \longrightarrow \infty} \varphi\left(\Re_{\mathfrak{n}}, \mathfrak{R}_{\mathfrak{n}+1}\right)=0
$$

Now, we claim that $\left\{\mathfrak{R}_{\mathfrak{n}}\right\}$ is a Cauchy. Assume that there are $\varepsilon>0$ and subsequences $\{\mathfrak{m}(j)\}$ and $\{\mathfrak{n}(j)\}$ so that

$\mathfrak{n}(j)>\mathfrak{m}(j)>j, \varphi\left(\mathfrak{R}_{\mathfrak{n}(j)}, \mathfrak{R}_{\mathfrak{m}(j)}\right) \geq \varepsilon$ and $\varphi\left(\mathfrak{R}_{\mathfrak{n}(j)}, \mathfrak{R}_{\mathfrak{m}(j)-1}\right)<\varepsilon$

Consider

$$
\begin{aligned}
\mathcal{E} \leq & \varphi\left(\mathfrak{R}_{\mathfrak{n}(j)}, \mathfrak{R}_{\mathfrak{m}(j)}\right) \leq \varphi\left(\mathfrak{R}_{\mathfrak{n}(j)}, \mathfrak{R}_{\mathfrak{m}(j)-1}\right) \\
& +\varphi\left(\mathfrak{R}_{\mathfrak{m}(j)-1}, \mathfrak{R}_{\mathfrak{m}(j)}\right)<\varepsilon+\varphi\left(\mathfrak{R}_{\mathfrak{m}(j)-1}, \Re_{\mathfrak{m}(j)}\right), j \in \mathbb{N}
\end{aligned}
$$

Taking the limit as $j \longrightarrow+\infty$ and using (8), we get

$$
\lim _{j \longrightarrow+\infty} \varphi\left(\mathfrak{R}_{\mathfrak{n}(j)}, \mathfrak{R}_{\mathfrak{m}(j)}\right)=\varepsilon
$$

Again,

$$
\begin{aligned}
\varphi\left(\mathfrak{R}_{\mathfrak{n}(j)}, \mathfrak{R}_{\mathfrak{m}(j)}\right) \leq & \left(\mathfrak{R}_{\mathfrak{m}(j)}, \mathfrak{R}_{\mathfrak{m}(j)+1}\right)+\varphi\left(\mathfrak{R}_{\mathfrak{m}(j)+1}, \mathfrak{R}_{\mathfrak{n}(j)+1}\right) \\
& +\varphi\left(\mathfrak{R}_{\mathfrak{n}(j)+1}, \mathfrak{R}_{\mathfrak{n}(j)}\right)
\end{aligned}
$$

$$
\begin{aligned}
\varphi\left(\mathfrak{R}_{\mathfrak{n}(j)+1}, \mathfrak{R}_{\mathfrak{m}(j)+1}\right) \leq & \varphi\left(\mathfrak{R}_{\mathfrak{m}(j)}, \mathfrak{R}_{\mathfrak{m}(j)+1}\right)+\varphi\left(\mathfrak{R}_{\mathfrak{m}(j)}, \mathfrak{R}_{\mathfrak{n}(j)}\right) \\
& +\varphi\left(\mathfrak{R}_{\mathfrak{n}(j)+1}, \mathfrak{R}_{\mathfrak{n}(j)}\right)
\end{aligned}
$$

Letting $j \longrightarrow+\infty$, together with (8) and (11), we deduce that

$$
\lim _{j \longrightarrow+\infty} \varphi\left(\mathfrak{R}_{\mathfrak{n}(j)+1}, \mathfrak{R}_{\mathfrak{m}(j)+1}\right)=\varepsilon
$$

Since there exists $\mathfrak{R}_{0} \in \mho$ such that $\mathfrak{R}_{0} \perp \mathfrak{G} \mathfrak{R}_{0}$ and $\alpha($ $\left.\mathfrak{R}_{0}, \mathfrak{G} \mathfrak{R}_{0}\right) \geq 1$, using condition (2), we derive that $\mathfrak{R}_{1} \perp \mathfrak{R}_{2}$, $\alpha\left(\mathfrak{R}_{1}, \mathfrak{R}_{2}\right)=\alpha\left(\mathfrak{G} \mathfrak{R}_{0}, \mathfrak{G}^{2} \mathfrak{R}_{0}\right) \geq 1$. By continuing this process, we get

$$
\mathfrak{R}_{\mathfrak{n}} \perp \mathfrak{R}_{\mathfrak{n}+1}, \alpha\left(\mathfrak{R}_{\mathfrak{n}}, \mathfrak{R}_{\mathfrak{n}+1}\right) \geq 1
$$

for all $\mathfrak{n} \geq 0$. Suppose that $\mathfrak{m}<\mathfrak{n}$. We have

$$
\begin{cases}\mathfrak{R}_{\mathfrak{m}} \perp \mathfrak{R}_{\mathfrak{m}+1}, & \alpha\left(\mathfrak{R}_{\mathfrak{m}}, \mathfrak{R}_{\mathfrak{m}+1}\right) \geq 1, \\ \mathfrak{R}_{\mathfrak{m}+1} \perp \mathfrak{R}_{\mathfrak{m}+2}, & \alpha\left(\mathfrak{R}_{\mathfrak{m}+1}, \mathfrak{R}_{\mathfrak{m}+2}\right) \geq 1 .\end{cases}
$$

Recall that $\mathfrak{G}$ is orthogonally triangular $\alpha$-admissible, so we have

$$
\mathfrak{R}_{\mathfrak{m}} \perp \mathfrak{R}_{\mathfrak{m}+2}, \alpha\left(\mathfrak{R}_{\mathfrak{m}}, \mathfrak{R}_{\mathfrak{m}+2}\right) \geq 1
$$

Again,

$$
\begin{cases}\mathfrak{R}_{\mathfrak{m}} \perp \mathfrak{R}_{\mathfrak{m}+2}, & \alpha\left(\mathfrak{R}_{\mathfrak{m}}, \mathfrak{R}_{\mathfrak{m}+2}\right) \geq 1, \\ \mathfrak{R}_{\mathfrak{m}+2} \perp \mathfrak{R}_{\mathfrak{m}+3}, & \alpha\left(\mathfrak{R}_{\mathfrak{m}+2}, \mathfrak{R}_{\mathfrak{m}+3}\right) \geq 1 .\end{cases}
$$

$\mathfrak{G}$ is orthogonally triangular $\alpha$-admissible, so we have

$$
\mathfrak{R}_{\mathfrak{m}} \perp \mathfrak{R}_{\mathfrak{m}+3}, \alpha\left(\mathfrak{R}_{\mathfrak{m}}, \mathfrak{R}_{\mathfrak{m}+3}\right) \geq 1
$$

By continuing this process, we get $\mathfrak{R}_{\mathfrak{m}} \perp \mathfrak{R}_{\mathfrak{n}}, \alpha\left(\mathfrak{R}_{\mathfrak{m}}, \mathfrak{R}_{\mathfrak{n}}\right.$ )$\geq 1$ and so $\mathfrak{R}_{\mathfrak{n}_{j}} \perp \mathfrak{R}_{\mathfrak{m}_{j}} \alpha\left(\mathfrak{R}_{\mathfrak{n}_{j}}, \mathfrak{R}_{\mathfrak{m}_{j}}\right) \geq 1$.

From (3), (11), and (14), we have

$$
\begin{aligned}
& \varphi\left(\mathfrak{R}_{\mathfrak{n}(j)+1}, \mathfrak{R}_{\mathfrak{m}(j)+1}\right)+\mathfrak{l} \\
& \leq\left(\varphi\left(\boldsymbol{R}_{\mathfrak{n}(j)+1}, \mathfrak{R}_{\mathfrak{m}(j)+1}\right)+\mathfrak{l}\right)^{\alpha\left(\Re_{\mathfrak{n}(j)}, \mathfrak{G} \Re_{\mathfrak{n}(j)}\right) \alpha\left(\Re_{\mathfrak{m}(j)}, \mathfrak{G} \Re_{\mathfrak{m}(j)}\right)} \\
& =\left(\varphi\left(\mathfrak{G} \Re_{\mathfrak{n}(j)}, \mathfrak{G} \mathfrak{R}_{\mathfrak{m}(j)}\right)+\mathfrak{l}\right)^{\alpha\left(\Re_{\mathfrak{n}(j)},\left(\mathfrak{G} \Re_{\mathfrak{n}(j)}\right) \alpha\left(\mathfrak{R}_{\mathfrak{m}(j)}, \mathfrak{G} \mathfrak{R}_{\mathfrak{m}(j)}\right)\right.} \\
& \leq \hbar\left(\varphi\left(\mathfrak{R}_{\mathfrak{n}(j)}, \mathfrak{R}_{\mathfrak{m}(j)}\right)\right) \varphi\left(\mathfrak{R}_{\mathfrak{n}(j)}, \mathfrak{R}_{\mathfrak{m}(j)}\right)+\mathfrak{l} .
\end{aligned}
$$

Hence,

$$
\frac{\varphi\left(\mathfrak{R}_{\mathfrak{n}(j)+1}, \mathfrak{R}_{\mathfrak{m}(j)+1}\right)}{\varphi\left(\mathfrak{R}_{\mathfrak{n}(j)}, \mathfrak{R}_{\mathfrak{m}(j)}\right)} \leq \hbar\left(\varphi\left(\mathfrak{R}_{\mathfrak{n}(j)}, \mathfrak{R}_{\mathfrak{m}(j)}\right)\right) \leq 1
$$

Letting $j \longrightarrow+\infty$ in the above inequality, we get

$$
\lim _{j \longrightarrow+\infty} \hbar\left(\varphi\left(\mathfrak{R}_{\mathfrak{n}(j)}, \mathfrak{R}_{\mathfrak{m}(j)}\right)\right)=1
$$

That is, at the limit $j \longrightarrow+\infty, \varphi\left(\mathfrak{R}_{\mathfrak{n}(j)}, \mathfrak{R}_{\mathfrak{m}(j)}\right)=0<\varepsilon$, which is a contradiction. Hence, $\left\{\mathfrak{R}_{\mathfrak{n}}\right\}$ is a Cauchy sequence in the complete metric space $\mho$, hence, there is $\mathscr{Z} \in \mho$ so that $\mathfrak{R}_{\mathfrak{n}} \longrightarrow \mathscr{Z}$. First, we suppose that $\mathfrak{G}$ is orthogonally continuous, then, we have

$$
\mathfrak{G} \mathscr{Z}=\lim _{\mathfrak{n} \longrightarrow \infty} \mathfrak{G} \mathfrak{R}_{\mathfrak{n}}=\lim _{\mathfrak{n} \longrightarrow \infty} \mathfrak{R}_{\mathfrak{n}+1}=\mathscr{Z}
$$

So, $\mathscr{Z}$ is a fixed point of $\mathfrak{G}$. Assume the condition (4) holds. Then, $\mathfrak{R}_{\mathfrak{n}} \perp \mathscr{Z}$ and $\alpha(\mathscr{Z}, \mathfrak{G} \mathscr{Z}) \geq 1$. Now, by (3), we 
have

$$
\begin{aligned}
\varphi\left(\mathfrak{G} \mathscr{Z}, \mathfrak{R}_{\mathfrak{n}+1}\right)+\mathfrak{l} & \leq\left(\varphi\left(\mathfrak{G} \mathscr{Z},\left(\mathfrak{G} \mathfrak{R}_{\mathfrak{n}}\right)+\mathfrak{l}\right)^{\alpha(\mathscr{E}, \mathfrak{G} \mathscr{Z}) \alpha\left(\Re_{\mathfrak{n}}, \mathfrak{G} \mathfrak{R}_{\mathfrak{n}}\right)}\right. \\
& \leq \hbar\left(\varphi\left(\mathscr{Z}, \mathfrak{R}_{\mathfrak{n}}\right)\right) \varphi\left(\mathscr{Z}, \mathfrak{R}_{\mathfrak{n}}\right)+\mathfrak{l} .
\end{aligned}
$$

That is, $\varphi\left(\mathfrak{G} \mathscr{Z}, \mathfrak{R}_{\mathfrak{n}+1}\right) \leq \hbar\left(\varphi\left(\mathscr{Z}, \mathfrak{R}_{\mathfrak{n}}\right)\right) \varphi\left(\mathscr{Z}, \mathfrak{R}_{\mathfrak{n}}\right)$, and so we get

$$
\begin{aligned}
\varphi(\mathfrak{G} \mathscr{Z}, \mathscr{Z}) & \leq \varphi\left(\mathfrak{G} \mathscr{Z}, \mathfrak{R}_{\mathfrak{n}+1}\right)+\varphi\left(\mathscr{Z}, \mathfrak{R}_{\mathfrak{n}+1}\right) \\
& \leq \hbar\left(\varphi\left(\mathscr{Z}, \mathfrak{R}_{\mathfrak{n}}\right)\right) \varphi\left(\mathscr{Z}, \mathfrak{R}_{\mathfrak{n}}\right)+\varphi\left(\mathscr{Z}, \mathfrak{R}_{\mathfrak{n}+1}\right) .
\end{aligned}
$$
(G).

Taking $\mathfrak{n} \longrightarrow \infty$, we find $\varphi(\mathfrak{G} \mathscr{Z}, \mathscr{Z})=0$, that is, $\mathscr{Z}=$

Theorem 8. Let $(\mho, \perp, \varphi)$ be an O-complete metric space. Given an orthogonal element $\mathfrak{R}_{0}$. The mappings $\mathfrak{G H}: \mho \longrightarrow$ $\mho$ and $\alpha: \mho \times \mho \longrightarrow[0, \infty)$ are such that

$$
\begin{aligned}
& \forall \mathfrak{R}, \mathcal{Y} \in \mho \text { with } \mathfrak{R} \perp \mathcal{Y}[\varphi(\mathfrak{G} \mathfrak{R}, \mathfrak{G} \mathscr{Y})>0 \Rightarrow \\
& \left.\cdot(\alpha(\mathfrak{R}, \mathfrak{G} \Re) \alpha(\mathscr{Y}, \mathfrak{G} \mathscr{Y})+1)^{\varphi(\mathfrak{G} \Re, \mathfrak{G})} \leq 2^{\hbar(\varphi(\mathfrak{R}, \mathscr{Y})) \varphi(\mathfrak{R}, \mathscr{Y})}\right],
\end{aligned}
$$

where $\mathfrak{l} \geq 1$. Assume that there exists a function $\hbar:[0, \infty)$ $\longrightarrow[0,1]$ such that, for any bounded sequence $\left\{\Omega_{\mathfrak{n}}\right\}$ of positive reals, $\hbar\left(\Omega_{\mathfrak{n}}\right) \longrightarrow 1$ implies $\Omega_{\mathfrak{n}} \longrightarrow 0$, satisfying the conditions:

(1) $\mathfrak{G}$ is $\perp$-preserving

(2) $\mathfrak{G}$ is orthogonally triangular $\alpha$-admissible

(3) There exists $\mathfrak{R}_{0} \in \mho$ such that $\mathfrak{R}_{0} \perp \mathfrak{G} \mathfrak{R}_{0}$ and $\alpha\left(\mathfrak{R}_{0}\right.$, $\left(\mathfrak{S} \Re_{0}\right) \geq 1$

(4) Either $\mathfrak{G}$ is orthogonally continuous, or if $\left\{\mathfrak{R}_{\mathfrak{n}}\right\}$ is a sequence in $\mho$ such that $\mathfrak{R}_{\mathfrak{n}} \longrightarrow \mathfrak{R}, \alpha\left(\mathfrak{R}_{\mathfrak{n}}, \mathfrak{R}_{\mathfrak{n}+1}\right) \geq$ 1 for all $\mathfrak{n}$, then $\mathfrak{R}_{\mathfrak{n}} \perp \mathfrak{R}$ and $\alpha(\mathfrak{R}, \mathfrak{G} \mathfrak{R}) \geq 1$

Then, $\mathfrak{G}$ has a fixed point.

Proof. By condition (3), there exists $\mathfrak{R}_{0} \in \mho$ such that $\mathfrak{R}_{0} \perp$ $\mathfrak{G} \mathfrak{R}_{0}$ and $\alpha\left(\mathfrak{R}_{0}, \mathfrak{G} \mathfrak{R}_{0}\right) \geq 1$. Let

$\mathfrak{R}_{1}:=\mathfrak{G} \mathfrak{R}_{0}, \mathfrak{R}_{2}:=\mathfrak{G} \mathfrak{R}_{1}=\mathfrak{G G}^{2} \mathfrak{R}_{0} \ldots \ldots, \mathfrak{R}_{n+1}:=\mathfrak{G} \mathfrak{R}_{n}=\mathfrak{G G}^{\mathfrak{n}+1} \mathfrak{R}_{0}$

for all $\mathfrak{n} \geq 0$. Since $\mathfrak{G}$ is $\perp$-preserving, $\left\{\mathfrak{R}_{n}\right\}$ is an $O$ -sequence in $\mho$. Condition (2) implies that $\alpha\left(\mathfrak{R}_{\mathfrak{n}}, \mathfrak{R}_{\mathfrak{n}+1}\right) \geq$ 1 for all $\mathfrak{n} \geq 0$. If $\mathfrak{R}_{\mathfrak{n}}=\mathfrak{R}_{\mathfrak{n}+1}$ for some $\mathfrak{n} \geq 0$, then $\mathfrak{R}_{\mathfrak{n}}$ is a fixed point of $\mathfrak{G}$. Assume that $\mathfrak{R}_{\mathfrak{n}} \neq \mathfrak{R}_{\mathfrak{n}+1}, \forall \mathfrak{n} \geq 0$. By Theorem 7 , we conclude that $\alpha\left(\mathfrak{R}_{\mathfrak{n}}, \mathfrak{G} \mathfrak{R}_{\mathfrak{n}}\right) \geq 1$ for all $\mathfrak{n} \geq 0$. From
(26), we obtain

$$
\begin{aligned}
2^{\varphi\left(\mathfrak{G} \Re_{\mathfrak{n}-1}, \mathfrak{G} \Re_{\mathfrak{n}}\right)} & \leq\left(\alpha\left(\boldsymbol{R}_{\mathfrak{n}-1}, \mathfrak{G} \Re_{\mathfrak{n}-1}\right) \alpha\left(\mathfrak{R}_{\mathfrak{n}}, \mathfrak{G} \Re_{\mathfrak{n}}\right)+1\right)^{\varphi\left(\mathfrak{G} \Re_{\mathfrak{n}-1}, \mathfrak{G} \Re_{\mathfrak{n}}\right)} \\
& \leq 2^{\hbar\left(\varphi\left(\Re_{\mathfrak{n}-1}, \Re_{\mathfrak{n}}\right)\right) \varphi\left(\Re_{\mathfrak{n}-1}, \mathfrak{R}_{\mathfrak{n}}\right)},
\end{aligned}
$$

which yields that

$$
\varphi\left(\mathfrak{R}_{\mathfrak{n}}, \mathfrak{R}_{\mathfrak{n}+1}\right) \leq \hbar\left(\varphi\left(\mathfrak{R}_{\mathfrak{n}-1}, \mathfrak{R}_{\mathfrak{n}}\right)\right) \varphi\left(\mathfrak{R}_{\mathfrak{n}-1}, \mathfrak{R}_{\mathfrak{n}}\right) .
$$

So, we conclude that $\varphi\left(\mathfrak{R}_{\mathfrak{n}}, \mathfrak{R}_{\mathfrak{n}+1}\right) \leq \varphi\left(\mathfrak{R}_{\mathfrak{n}-1}, \mathfrak{R}_{\mathfrak{n}}\right)$. It follows that the sequence $\mathfrak{d}_{\mathfrak{n}}:=\varphi\left(\mathfrak{R}_{\mathfrak{n}}, \mathfrak{R}_{\mathfrak{n}+1}\right)$ is decreasing, so there is $\mathfrak{d} \geq 0$ so that $\mathfrak{d}_{\mathfrak{n}} \longrightarrow \mathfrak{d}$ as $\mathfrak{n} \longrightarrow \infty$. We claim that $\mathfrak{d}=0$. Assume that $\mathfrak{d}>0$. Considering (29), we have

$$
\frac{\varphi\left(\boldsymbol{R}_{\mathfrak{n}}, \mathfrak{R}_{\mathfrak{n}+1}\right)}{\varphi\left(\boldsymbol{R}_{\mathfrak{n}-1}, \mathfrak{R}_{\mathfrak{n}}\right)} \leq \hbar\left(\varphi\left(\boldsymbol{R}_{\mathfrak{n}-1}, \mathfrak{R}_{\mathfrak{n}}\right)\right) \leq 1,
$$

which implies $\lim _{\mathfrak{n} \longrightarrow \infty} \hbar\left(\varphi\left(\Re_{\mathfrak{n}-1}, \Re_{\mathfrak{n}}\right)\right)=1$.

Therefore, $\mathfrak{d}=\lim _{\mathfrak{n} \longrightarrow \infty} \mathfrak{d}_{\mathfrak{n}}=\lim _{\mathfrak{n} \longrightarrow \infty} \varphi\left(\mathfrak{R}_{\mathfrak{n}-1}, \mathfrak{R}_{\mathfrak{n}}\right)=0$. It is a contradiction. Thus,

$$
\lim _{\mathfrak{n} \longrightarrow \infty} \varphi\left(\mathfrak{R}_{\mathfrak{n}}, \mathfrak{R}_{\mathfrak{n}+1}\right)=0 .
$$

Now, we claim that $\left\{\mathfrak{R}_{\mathfrak{n}}\right\}$ is a Cauchy sequence. Assume there are $\varepsilon>0$ and sequences $\{\mathfrak{m}(j)\}$ and $\{\mathfrak{n}(j)\}$ so that

$\mathfrak{n}(j)>\mathfrak{m}(j)>j, \varphi\left(\mathfrak{R}_{\mathfrak{n}(j)}, \mathfrak{R}_{\mathfrak{m}(j)}\right) \geq \varepsilon$ and $\varphi\left(\mathfrak{R}_{\mathfrak{n}(j)}, \mathfrak{R}_{\mathfrak{m}(j)-1}\right)<\varepsilon$

As in the proof of Theorem 7, one writes

$$
\begin{array}{r}
\lim _{j \longrightarrow+\infty} \varphi\left(\mathfrak{R}_{\mathfrak{n}(j)}, \mathfrak{R}_{\mathfrak{m}(j)}\right)=\varepsilon, \\
\lim _{j \longrightarrow+\infty} \varphi\left(\mathfrak{R}_{\mathfrak{n}(j)+1}, \mathfrak{R}_{\mathfrak{m}(j)+1}\right)=\varepsilon .
\end{array}
$$

Since there exists $\mathfrak{R}_{0} \in \mho$ such that $\mathfrak{R}_{0} \perp \mathfrak{G} \mathfrak{R}_{0}$ and $\alpha($ $\left.\mathfrak{R}_{0}, \mathfrak{G} \mathfrak{R}_{0}\right) \geq 1$, using condition (2), we derive that

$$
\mathfrak{R}_{1} \perp \mathfrak{R}_{2}, \alpha\left(\mathfrak{R}_{1}, \mathfrak{R}_{2}\right)=\alpha\left(\mathfrak{G} \Re_{0}, \mathfrak{G}^{2} \mathfrak{R}_{0}\right) \geq 1 .
$$

By continuing this process, we get

$$
\mathfrak{R}_{\mathfrak{n}} \perp \mathfrak{R}_{\mathfrak{n}+1}, \alpha\left(\mathfrak{R}_{\mathfrak{n}}, \mathfrak{R}_{\mathfrak{n}+1}\right) \geq 1,
$$

for all $\mathfrak{n} \geq 0$. Suppose that $\mathfrak{m}<\mathfrak{n}$. Recall that

$$
\begin{cases}\mathfrak{R}_{\mathfrak{m}} \perp \mathfrak{R}_{\mathfrak{m}+1}, & \alpha\left(\mathfrak{R}_{\mathfrak{m}}, \mathfrak{R}_{\mathfrak{m}+1}\right) \geq 1, \\ \mathfrak{R}_{\mathfrak{m}+1} \perp \mathfrak{R}_{\mathfrak{m}+2}, & \alpha\left(\mathfrak{R}_{\mathfrak{m}+1}, \mathfrak{R}_{\mathfrak{m}+2}\right) \geq 1 .\end{cases}
$$

Since $\mathfrak{G}$ is orthogonally triangular $\alpha$-admissible, we have

$$
\mathfrak{R}_{\mathfrak{m}} \perp \mathfrak{R}_{\mathfrak{m}+2}, \alpha\left(\mathfrak{R}_{\mathfrak{m}}, \mathfrak{R}_{\mathfrak{m}+2}\right) \geq 1 .
$$


Again,

$$
\begin{cases}\mathfrak{R}_{\mathfrak{m}} \perp \mathfrak{R}_{\mathfrak{m}+2}, & \alpha\left(\mathfrak{R}_{\mathfrak{m}}, \mathfrak{R}_{\mathfrak{m}+2}\right) \geq 1, \\ \mathfrak{R}_{\mathfrak{m}+2} \perp \mathfrak{R}_{\mathfrak{m}+3}, & \alpha\left(\mathfrak{R}_{\mathfrak{m}+2}, \mathfrak{R}_{\mathfrak{m}+3}\right) \geq 1 .\end{cases}
$$

(G) is orthogonally triangular $\alpha$-admissible, so

$$
\mathfrak{R}_{\mathfrak{m}} \perp \mathfrak{R}_{\mathfrak{m}+3}, \alpha\left(\mathfrak{R}_{\mathfrak{m}}, \mathfrak{R}_{\mathfrak{m}+3}\right) \geq 1 .
$$

By continuing this process, we get $\mathfrak{R}_{\mathfrak{m}} \perp \mathfrak{R}_{\mathfrak{n}}, \alpha\left(\boldsymbol{R}_{\mathfrak{m}}, \mathfrak{R}_{\mathfrak{n}}\right.$ )$\geq 1$ and so $\mathfrak{R}_{\mathfrak{n}_{j}} \perp \mathfrak{R}_{\mathfrak{m}_{j}} \alpha\left(\mathfrak{R}_{\mathfrak{n}_{j}}, \mathfrak{R}_{\mathfrak{m}_{j}}\right) \geq 1$.

Now, from (26), (33), and (34), we have

$$
\begin{aligned}
& 2^{\varphi\left(\Re_{\mathfrak{n}(j)+1}, \Re_{\mathfrak{m}(j)+1}\right)} \leq\left(\alpha \left(\mathfrak{R}_{\mathfrak{n}(j)},\left(\mathfrak{S} \Re_{\mathfrak{n}(j)}\right) \alpha\right.\right. \\
& \left.\cdot\left(\mathfrak{R}_{\mathfrak{m}(j)}, \mathfrak{G} \mathfrak{R}_{\mathfrak{m}(j)}\right)+1\right)^{\varphi\left(\Re_{\mathfrak{n}(j)+1}, \Re_{\mathfrak{m}(j)+1}\right)} \\
& =\left(\alpha\left(\mathfrak{R}_{\mathfrak{n}(j)}, \mathfrak{G} \mathfrak{R}_{\mathfrak{n}(j)}\right)\right. \\
& \left.\cdot \alpha\left(\mathfrak{R}_{\mathfrak{m}(j)}, \mathfrak{G} \Re_{\mathfrak{m}(j)}\right)+1\right)^{\varphi\left(\mathfrak{G} \Re_{\mathfrak{n}(j)}, \mathfrak{G} \Re_{\mathfrak{m}(j)}\right)} \\
& \leq 2^{\hbar\left(\varphi\left(\Re_{\mathfrak{n}(j)}, \Re_{\mathfrak{m}(j)}\right)\right) \varphi\left(\Re_{\mathfrak{n}(j)}, \Re_{\mathfrak{m}(j)}\right) .}
\end{aligned}
$$

Hence,

$$
\frac{\varphi\left(\mathfrak{R}_{\mathfrak{n}(j)+1}, \mathfrak{R}_{\mathfrak{m}(j)+1}\right)}{\varphi\left(\boldsymbol{R}_{\mathfrak{n}(j)}, \mathfrak{R}_{\mathfrak{m}(j)}\right)} \leq \hbar\left(\varphi\left(\mathfrak{R}_{\mathfrak{n}(j)}, \mathfrak{R}_{\mathfrak{m}(j)}\right)\right) \leq 1 .
$$

Letting $j \longrightarrow+\infty$, we get

$$
\lim _{j \longrightarrow+\infty} \hbar\left(\varphi\left(\mathfrak{R}_{\mathfrak{n}(j)}, \mathfrak{R}_{\mathfrak{m}(j)}\right)\right)=1 .
$$

That is, $\lim _{j \longrightarrow+\infty} \varphi\left(\mathfrak{R}_{\mathfrak{n}(j)}, \mathfrak{R}_{\mathfrak{m}(j)}\right)=0<\varepsilon$. It is a contradiction, so $\left\{\Re_{\mathfrak{n}}\right\}$ is a Cauchy sequence, hence, there is $\mathscr{Z}$ $\in \mho$ so that $\mathfrak{R}_{\mathfrak{n}} \longrightarrow \mathscr{Z}$. First, assume that $\mathfrak{G}$ is orthogonally continuous, then

$$
\mathfrak{G} \mathscr{Z}=\lim _{\mathfrak{n} \longrightarrow \infty} \mathfrak{G} \Re_{\mathfrak{n}}=\lim _{\mathfrak{n} \longrightarrow \infty} \mathfrak{G} \mathfrak{R}_{\mathfrak{n}+1}=\mathscr{Z} .
$$

So, $\mathscr{Z}$ is a fixed point of $\mathfrak{G}$. If (4) is verified, so $\mathfrak{R}_{\mathfrak{n}} \perp \mathscr{Z}$ and $\alpha(\mathscr{Z}, \mathfrak{G} \mathscr{Z}) \geq 1$. Now, by (26), we have

$$
\begin{aligned}
2^{\varphi\left(\mathfrak{G} \mathscr{Z}, \Re_{\mathfrak{n}+1}\right)} & \leq\left(\alpha(\mathscr{Z}, \mathfrak{G} \mathscr{Z}) \alpha\left(\mathfrak{R}_{\mathfrak{n}},\left(\mathfrak{G} \Re_{\mathfrak{n}}\right)+1\right)^{\varphi\left(\mathfrak{G} \mathscr{E}, \mathfrak{G} \mathfrak{R}_{\mathfrak{n}}\right)}\right. \\
& \leq 2^{\hbar\left(\varphi\left(\mathscr{X}, \Re_{\mathfrak{n}}\right)\right) \varphi\left(\mathscr{E}, \Re_{\mathfrak{n}}\right)} .
\end{aligned}
$$

That is, $\varphi\left(\mathfrak{G} \mathscr{Z}, \mathfrak{R}_{\mathfrak{n}+1}\right) \leq \hbar\left(\varphi\left(\mathscr{Z}, \mathfrak{R}_{\mathfrak{n}}\right)\right) \varphi\left(\mathscr{Z}, \mathfrak{R}_{\mathfrak{n}}\right)$, and so we get

$$
\begin{aligned}
\varphi(\mathfrak{G} \mathscr{Z}, \mathscr{Z}) & \leq \varphi\left(\mathfrak{G} \mathscr{Z}, \mathfrak{R}_{\mathfrak{n}+1}\right)+\varphi\left(\mathscr{Z}, \mathfrak{R}_{\mathfrak{n}+1}\right) \\
& \leq \hbar\left(\varphi\left(\mathscr{Z}, \mathfrak{R}_{\mathfrak{n}}\right)\right) \varphi\left(\mathscr{Z}, \mathfrak{R}_{\mathfrak{n}}\right)+\varphi\left(\mathscr{Z}, \mathfrak{R}_{\mathfrak{n}+1}\right) .
\end{aligned}
$$

At the limit $\mathfrak{n} \longrightarrow \infty$, one gets $\varphi(\mathfrak{G} \mathscr{Z}, \mathscr{Z})=0$, that is, $\mathscr{Z}=\mathfrak{G} \mathscr{Z}$.

Theorem 9. Let $(\mho, \perp, \varphi)$ be an O-complete metric space. Given an orthogonal element $\mathfrak{R}_{0}$. The mappings $\mathfrak{G}: \mho \longrightarrow$ $\mho$ and $\alpha: \mho \times \mho \longrightarrow[0, \infty)$ are such that

$$
\begin{aligned}
& \forall \mathfrak{R}, \mathscr{Y} \in \mho \text { with } \mathfrak{R} \perp \mathcal{Y}[\varphi(\mathfrak{G} \mathfrak{R}, \mathfrak{G} \mathcal{Y}) \\
& \quad>0 \Rightarrow \alpha(\mathfrak{R}, \mathfrak{G} \mathfrak{R}) \alpha(\mathcal{Y}, \mathfrak{G} \mathscr{Y}) \varphi(\mathfrak{G} \mathfrak{R}, \mathfrak{G} \mathscr{Y}) \\
& \quad \leq \hbar(\varphi(\mathfrak{R}, \mathcal{Y})) \varphi(\mathfrak{R}, \mathcal{Y})],
\end{aligned}
$$

where $\mathfrak{l} \geq 1$. Assume that there exists a function $\hbar:[0, \infty)$ $\longrightarrow[0,1]$ such that, for any bounded sequence $\left\{\Omega_{\mathfrak{n}}\right\}$ of positive reals, $\hbar\left(\Omega_{\mathfrak{n}}\right) \longrightarrow 1$ implies $\Omega_{\mathfrak{n}} \longrightarrow 0$, satisfying the conditions:

(1) $\mathfrak{G}$ is $\perp$-preserving

(2) $\mathfrak{G}$ is orthogonally triangular $\alpha$-admissible

(3) There exists $\mathfrak{R}_{0} \in \mho$ such that $\mathfrak{R}_{0} \perp \mathfrak{G} \mathfrak{R}_{0}$ and $\alpha\left(\mathfrak{R}_{0}\right.$, $\left(\mathfrak{G} \mathfrak{R}_{0}\right) \geq 1$

(4) Either $\mathbb{G}$ is orthogonally continuous, or if $\left\{\mathfrak{R}_{\mathfrak{n}}\right\}$ is a sequence in $\mho$ such that $\mathfrak{R}_{\mathfrak{n}} \longrightarrow \mathfrak{R}, \alpha\left(\mathfrak{R}_{\mathfrak{n}}, \mathfrak{R}_{\mathfrak{n}+1}\right) \geq$ 1 for all $\mathfrak{n}$, then $\mathfrak{R}_{\mathfrak{n}} \perp \mathfrak{R}$ and $\alpha(\mathfrak{R}, \mathfrak{G} \Re) \geq 1$

Then, $\mathfrak{G}$ has a fixed point.

Proof. By condition (3), there exists $\mathfrak{R}_{0} \in \mho$ such that $\mathfrak{R}_{0} \perp$ $\mathfrak{G} \mathfrak{R}_{0}$ and $\alpha\left(\mathfrak{R}_{0}, \mathfrak{G} \Re_{0}\right) \geq 1$. Let

$$
\mathfrak{R}_{1}:=\mathfrak{G} \mathfrak{R}_{0}, \mathfrak{R}_{2}:=\mathfrak{G} \mathfrak{R}_{1}=\mathfrak{G G}^{2} \mathfrak{R}_{0}, \cdots, \mathfrak{R}_{n+1}:=\mathfrak{G} \mathfrak{R}_{n}=\left(\mathfrak{G}^{\mathfrak{n}+1} \mathfrak{R}_{0}\right.
$$

for all $\mathfrak{n} \geq 0$. Since $\mathfrak{G}$ is $\perp$-preserving, $\left\{\mathfrak{R}_{n}\right\}$ is an $O$ -sequence in $\mho$. Condition (2) yields that $\alpha\left(\mathfrak{R}_{\mathfrak{n}}, \mathfrak{R}_{\mathfrak{n}+1}\right) \geq 1$ for all $\mathfrak{n} \geq 0$. If $\mathfrak{R}_{\mathfrak{n}}=\mathfrak{R}_{\mathfrak{n}+1}$ for any $\mathfrak{n} \geq 0$, then $\mathfrak{R}_{\mathfrak{n}}$ is a fixed point of $\mathfrak{G}$. Assume that $\mathfrak{R}_{\mathfrak{n}} \neq \mathfrak{R}_{\mathfrak{n}+1}, \forall \mathfrak{n} \geq 0$. By Theorem 7, we conclude that $\alpha\left(\mathfrak{R}_{\mathfrak{n}}, \mathfrak{G} \mathfrak{R}_{\mathfrak{n}}\right) \geq 1$ for all $\mathfrak{n} \geq 0$. From (47), we obtain

$$
\begin{aligned}
& \alpha\left(\Re_{\mathfrak{n}-1}, \mathfrak{G} \Re_{\mathfrak{n}-1}\right) \alpha\left(\mathfrak{R}_{\mathfrak{n}},\left(\mathfrak{G} \Re_{\mathfrak{n}}\right) \varphi\left(\mathfrak{G} \Re_{\mathfrak{n}-1},\left(\mathfrak{G} \Re_{\mathfrak{n}}\right)\right.\right. \\
& \quad \leq \hbar\left(\varphi\left(\boldsymbol{R}_{\mathfrak{n}-1}, \mathfrak{R}_{\mathfrak{n}}\right)\right) \varphi\left(\mathfrak{R}_{\mathfrak{n}-1}, \mathfrak{R}_{\mathfrak{n}}\right),
\end{aligned}
$$

then

$$
\varphi\left(\boldsymbol{R}_{\mathfrak{n}}, \mathfrak{R}_{\mathfrak{n}+1}\right) \leq \hbar\left(\varphi\left(\boldsymbol{R}_{\mathfrak{n}-1}, \mathfrak{R}_{\mathfrak{n}}\right)\right) \varphi\left(\boldsymbol{R}_{\mathfrak{n}-1}, \mathfrak{R}_{\mathfrak{n}}\right)
$$

So, we conclude that $\varphi\left(\Re_{\mathfrak{n}}, \mathfrak{R}_{\mathfrak{n}+1}\right) \leq \varphi\left(\mathfrak{R}_{\mathfrak{n}-1}, \mathfrak{R}_{\mathfrak{n}}\right)$. Thus, $\left\{\varphi\left(\mathfrak{R}_{\mathfrak{n}}, \mathfrak{R}_{\mathfrak{n}+1}\right)\right\}$ is decreasing; hence, there is $\mathfrak{D} \geq 0$ so that $\varphi\left(\mathfrak{R}_{\mathfrak{n}}, \mathfrak{R}_{\mathfrak{n}+1}\right) \longrightarrow \mathfrak{d}$ as $\mathfrak{n} \longrightarrow \infty$. Regarding (50), we obtain

$$
\frac{\varphi\left(\mathfrak{R}_{\mathfrak{n}}, \mathfrak{R}_{\mathfrak{n}+1}\right)}{\varphi\left(\mathfrak{R}_{\mathfrak{n}-1}, \mathfrak{R}_{\mathfrak{n}}\right)} \leq \hbar\left(\varphi\left(\boldsymbol{R}_{\mathfrak{n}-1}, \mathfrak{R}_{\mathfrak{n}}\right)\right) \leq 1
$$

which implies that $\lim _{\mathfrak{n} \longrightarrow \infty} \hbar\left(\varphi\left(\mathfrak{R}_{\mathfrak{n}-1}, \mathfrak{R}_{\mathfrak{n}}\right)\right)=1$. Hence, $\mathfrak{d}=\lim _{\mathfrak{n} \longrightarrow \infty} \mathfrak{d}_{\mathfrak{n}}=\lim _{\mathfrak{n} \longrightarrow \infty} \varphi\left(\mathfrak{R}_{\mathfrak{n}-1}, \mathfrak{R}_{\mathfrak{n}}\right)=0$, which is a 
contradiction. Hence, we derive that

$$
\lim _{\mathfrak{n} \longrightarrow \infty} \varphi\left(\Re_{\mathfrak{n}}, \Re_{\mathfrak{n}+1}\right)=0
$$

We claim that $\left\{\mathfrak{R}_{\mathfrak{n}}\right\}$ is Cauchy sequence. Assume that there are $\varepsilon>0$ and subsequences $\{\mathfrak{m}(j)\}$ and $\{\mathfrak{n}(j)\}$ so that

$\mathfrak{n}(j)>\mathfrak{m}(j)>j, \varphi\left(\mathfrak{R}_{\mathfrak{n}(j)}, \mathfrak{R}_{\mathfrak{m}(j)}\right) \geq \varepsilon$ and $\varphi\left(\mathfrak{R}_{\mathfrak{n}(j)}, \mathfrak{R}_{\mathfrak{m}(j)-1}\right)<\varepsilon$

As in Theorem 7, one has

$$
\begin{array}{r}
\lim _{j \longrightarrow+\infty} \varphi\left(\mathfrak{R}_{\mathfrak{n}(j)}, \mathfrak{R}_{\mathfrak{m}(j)}\right)=\varepsilon, \\
\lim _{j \longrightarrow+\infty} \varphi\left(\mathfrak{R}_{\mathfrak{n}(j)+1}, \mathfrak{R}_{\mathfrak{m}(j)+1}\right)=\varepsilon .
\end{array}
$$

Since there exists $\mathfrak{R}_{0} \in \mho$ such that $\mathfrak{R}_{0} \perp \mathfrak{G} \Re_{0}$ and $\alpha($ $\left.\mathfrak{R}_{0}, \mathfrak{G} \mathfrak{R}_{0}\right) \geq 1$, using condition (2), we derive that

$$
\mathfrak{R}_{1} \perp \mathfrak{R}_{2}, \alpha\left(\mathfrak{R}_{1}, \mathfrak{R}_{2}\right)=\alpha\left(\mathfrak{G} \mathfrak{R}_{0}, \mathfrak{G}^{2} \mathfrak{R}_{0}\right) \geq 1
$$

By continuing this process, we get

$$
\mathfrak{R}_{\mathfrak{n}} \perp \mathfrak{R}_{\mathfrak{n}+1}, \alpha\left(\mathfrak{R}_{\mathfrak{n}}, \mathfrak{R}_{\mathfrak{n}+1}\right) \geq 1
$$

for all $\mathfrak{n} \geq 0$. Suppose that $\mathfrak{m}<\mathfrak{n}$. Recall that

$$
\begin{cases}\mathfrak{R}_{\mathfrak{m}} \perp \mathfrak{R}_{\mathfrak{m}+1}, & \alpha\left(\mathfrak{R}_{\mathfrak{m}}, \mathfrak{R}_{\mathfrak{m}+1}\right) \geq 1, \\ \mathfrak{R}_{\mathfrak{m}+1} \perp \mathfrak{R}_{\mathfrak{m}+2}, & \alpha\left(\mathfrak{R}_{\mathfrak{m}+1}, \mathfrak{R}_{\mathfrak{m}+2}\right) \geq 1 .\end{cases}
$$

Since $\mathfrak{G}$ is orthogonally triangular $\alpha$-admissible, we have

$$
\mathfrak{R}_{\mathfrak{m}} \perp \mathfrak{R}_{\mathfrak{m}+2}, \alpha\left(\mathfrak{R}_{\mathfrak{m}}, \mathfrak{R}_{\mathfrak{m}+2}\right) \geq 1
$$

Again, since

$$
\begin{cases}\mathfrak{R}_{\mathfrak{m}} \perp \mathfrak{R}_{\mathfrak{m}+2}, & \alpha\left(\mathfrak{R}_{\mathfrak{m}}, \mathfrak{R}_{\mathfrak{m}+2}\right) \geq 1, \\ \mathfrak{R}_{\mathfrak{m}+2} \perp \mathfrak{R}_{\mathfrak{m}+3}, & \alpha\left(\mathfrak{R}_{\mathfrak{m}+2}, \mathfrak{R}_{\mathfrak{m}+3}\right) \geq 1,\end{cases}
$$

using the fact that $\mathfrak{G}$ is orthogonally triangular $\alpha$-admissible, we have

$$
\mathfrak{R}_{\mathfrak{m}} \perp \mathfrak{R}_{\mathfrak{m}+3}, \alpha\left(\Re_{\mathfrak{m}}, \mathfrak{R}_{\mathfrak{m}+3}\right) \geq 1 .
$$

By continuing this process, we get $\mathfrak{R}_{\mathfrak{m}} \perp \mathfrak{R}_{\mathfrak{n}}, \alpha\left(\mathfrak{R}_{\mathfrak{m}}, \mathfrak{R}_{\mathfrak{n}}\right.$ )$\geq 1$ and so $\mathfrak{R}_{\mathfrak{n}_{j}} \perp \mathfrak{R}_{\mathfrak{m}_{j}} \alpha\left(\mathfrak{R}_{\mathfrak{n}_{j}}, \mathfrak{R}_{\mathfrak{m}_{j}}\right) \geq 1$.
Now, from (47), (54), and (55), we have

$$
\begin{aligned}
\varphi\left(\mathfrak{R}_{\mathfrak{n}(j)+1}, \mathfrak{R}_{\mathfrak{m}(j)+1}\right) \leq & \alpha\left(\mathfrak{R}_{\mathfrak{n}(j)}, \mathfrak{G} \Re_{\mathfrak{n}(j)}\right) \alpha\left(\mathfrak{R}_{\mathfrak{m}(j)}, \mathfrak{G} \Re_{\mathfrak{m}(j)}\right) \varphi \\
& \cdot\left(\mathfrak{R}_{\mathfrak{n}(j)+1}, \mathfrak{R}_{\mathfrak{m}(j)+1}\right)=\alpha\left(\mathfrak{R}_{\mathfrak{n}(j)}, \mathfrak{G} \mathfrak{R}_{\mathfrak{n}(j)}\right) \alpha \\
& \cdot\left(\mathfrak{R}_{\mathfrak{m}(j)}, \mathfrak{G} \mathfrak{R}_{\mathfrak{m}(j)}\right) \varphi\left(\mathfrak{G} \mathfrak{R}_{\mathfrak{n}(j)}, \mathfrak{G} \mathfrak{R}_{\mathfrak{m}(j)}\right) \\
\leq & \hbar\left(\varphi\left(\mathfrak{R}_{\mathfrak{n}(j)}, \mathfrak{R}_{\mathfrak{m}(j)}\right)\right) \varphi\left(\mathfrak{R}_{\mathfrak{n}(j)}, \mathfrak{R}_{\mathfrak{m}(j)}\right) .
\end{aligned}
$$

Hence,

$$
\frac{\varphi\left(\mathfrak{R}_{\mathfrak{n}(j)+1}, \mathfrak{R}_{\mathfrak{m}(j)+1}\right)}{\varphi\left(\mathfrak{R}_{\mathfrak{n}(j)}, \mathfrak{R}_{\mathfrak{m}(j)}\right)} \leq \hbar\left(\varphi\left(\mathfrak{R}_{\mathfrak{n}(j)}, \mathfrak{R}_{\mathfrak{m}(j)}\right)\right) \leq 1
$$

Letting $j \longrightarrow+\infty$, we get

$$
\lim _{j \longrightarrow+\infty} \hbar\left(\varphi\left(\mathfrak{R}_{\mathfrak{n}(j)}, \mathfrak{R}_{\mathfrak{m}(j)}\right)\right)=1
$$

At the limit $j \longrightarrow+\infty, \varphi\left(\mathfrak{R}_{\mathfrak{n}(j)}, \mathfrak{R}_{\mathfrak{m}(j)}\right)=0$. Hence, $\left\{\mathfrak{R}_{\mathfrak{n}}\right\}$ is a Cauchy sequence. The completness of $\mho$ ensures that there is $\mathscr{Z} \in \mho$ so that $\mathfrak{R}_{\mathfrak{n}} \longrightarrow \mathscr{Z}$. If $\mathfrak{G}$ is orthogonally continuous, then

$$
\mathfrak{G} \mathscr{Z}=\lim _{\mathfrak{n} \longrightarrow \infty} \mathfrak{G} \mathfrak{R}_{\mathfrak{n}}=\lim _{\mathfrak{n} \longrightarrow \infty} \mathfrak{G} \Re_{\mathfrak{n}+1}=\mathscr{Z} .
$$

So, $\mathscr{Z}$ is a fixed point of $\mathfrak{G}$. If (4) is verified, so $\mathfrak{R}_{\mathfrak{n}} \perp \mathscr{Z}$ and $\alpha(\mathscr{Z}, \mathfrak{G} \mathscr{Z}) \geq 1$. Now, by (47), we have

$$
\begin{aligned}
\varphi\left(\mathfrak{G} \mathscr{Z}, \mathfrak{R}_{\mathfrak{n}+1}\right) & \leq \alpha(\mathscr{Z}, \mathfrak{G} \mathscr{Z}) \alpha\left(\mathfrak{R}_{\mathfrak{n}}, \mathfrak{G} \mathfrak{R}_{\mathfrak{n}}\right) \varphi\left(\mathfrak{G} \mathscr{Z}, \mathfrak{G} \mathfrak{R}_{\mathfrak{n}}\right) \\
& \leq \hbar\left(\varphi\left(\mathscr{Z}, \mathfrak{R}_{\mathfrak{n}}\right)\right) \varphi\left(\mathscr{Z}, \mathfrak{R}_{\mathfrak{n}}\right) .
\end{aligned}
$$

That is, $\varphi\left(\mathfrak{G} \mathscr{Z}, \mathfrak{R}_{\mathfrak{n}+1}\right) \leq \hbar\left(\varphi\left(\mathscr{Z}, \mathfrak{R}_{\mathfrak{n}}\right)\right) \varphi\left(\mathscr{Z}, \mathfrak{R}_{\mathfrak{n}}\right)$, and so we get

$$
\begin{aligned}
\varphi(\mathfrak{G} \mathscr{Z}, \mathscr{Z}) & \leq \varphi\left(\mathfrak{G} \mathscr{Z}, \mathfrak{R}_{\mathfrak{n}+1}\right)+\varphi\left(\mathscr{Z}, \mathfrak{R}_{\mathfrak{n}+1}\right) \\
& \leq \hbar\left(\varphi\left(\mathscr{Z}, \mathfrak{R}_{\mathfrak{n}}\right)\right) \varphi\left(\mathscr{Z}, \mathfrak{R}_{\mathfrak{n}}\right)+\varphi\left(\mathscr{Z}, \mathfrak{R}_{\mathfrak{n}+1}\right) .
\end{aligned}
$$
$\mathfrak{G} \mathscr{Z}$.

Taking $\mathfrak{n} \longrightarrow \infty$, one has $\varphi(\mathfrak{G} \mathscr{Z}, \mathscr{Z})=0$, that is, $\mathscr{Z}=$

Theorem 10. Assume that all the hypotheses of Theorems 7, 8, and 9 hold. Adding the following condition:

(c) If $\mathfrak{R}=\mathfrak{G} \Re$ and $\mathscr{Z}=\mathfrak{G} \mathscr{Z}$ then $\mathfrak{R} \perp \mathscr{Z}, \alpha(\mathfrak{R}, \mathfrak{G} \mathfrak{R}) \geq 1$ and $\alpha(\mathscr{Z}, \mathfrak{G} \mathscr{Z}) \geq 1$, we obtain the uniqueness of the fixed point of $\mathfrak{G}$.

Proof. Suppose that $\mathscr{Z}$ and $\mathscr{Z}^{*}$ are two fixed points of $\mathscr{H}$ such that $\mathscr{Z} \neq \mathscr{Z}^{*}$. Then, $\mathscr{Z} \perp \mathscr{Z}^{*} \alpha(\mathscr{Z}, \mathfrak{G}) \geq 1$ and $\alpha\left(\mathscr{Z}^{*}\right.$, 
$\left(\mathfrak{G} \mathscr{Z}^{*}\right) \geq 1$. For Theorem 7 , we have

$$
\begin{aligned}
\varphi\left(\mathfrak{G} \mathscr{Z}, \mathfrak{G} \mathscr{Z}^{*}\right)+\mathfrak{l} & \leq\left(\varphi\left(\mathfrak{G} \mathscr{Z}, \mathfrak{G} \mathscr{Z}^{*}\right)+\mathfrak{l}\right)^{\alpha(\mathscr{E}, \mathfrak{G} \mathscr{Z}) \alpha\left(\mathscr{Z}^{*}, \mathfrak{G} \mathscr{Z}^{*}\right)} \\
& \leq \hbar\left(\varphi\left(\mathscr{Z}, \mathscr{Z}^{*}\right)\right) \varphi\left(\mathscr{Z}, \mathscr{Z}^{*}\right)+\mathfrak{l} .
\end{aligned}
$$

For Theorem 8, we have

$$
\begin{aligned}
2^{\varphi\left(\mathfrak{G} \mathscr{Z}, \mathfrak{G} \mathscr{I}^{*}\right)} & \leq\left(\alpha(\mathscr{Z}, \mathfrak{G} \mathscr{Z}) \alpha\left(\mathscr{Z}^{*},\left(\mathfrak{G} \mathscr{Z}^{*}\right)+1\right)^{\varphi\left(\mathfrak{G} \mathscr{Z}, \mathfrak{G} \mathscr{E}^{*}\right)}\right. \\
& \leq 2^{\hbar\left(\varphi\left(\mathscr{Z}, \mathscr{E}^{*}\right)\right) \varphi\left(\mathscr{Z}, \mathscr{E}^{*}\right)} .
\end{aligned}
$$

For Theorem 9, we have

$$
\begin{aligned}
\varphi\left(\mathfrak{G} \mathscr{Z}, \mathfrak{G} \mathscr{Z}^{*}\right) & \leq \alpha(\mathscr{Z}, \mathfrak{G} \mathscr{Z}) \alpha\left(\mathscr{Z}^{*}, \mathfrak{G} \mathscr{Z}^{*}\right) \varphi\left(\mathfrak{G} \mathscr{Z}, \mathfrak{G}^{*}\right) \\
& \leq \hbar\left(\varphi\left(\mathscr{Z}, \mathscr{Z}^{*}\right)\right) \varphi\left(\mathscr{Z}, \mathscr{Z}^{*}\right) .
\end{aligned}
$$

We deduce that $\hbar\left(\varphi\left(\mathscr{Z}, \mathscr{Z}^{*}\right)\right)=1$, and so $\varphi\left(\mathscr{Z}, \mathscr{Z}^{*}\right)=0$. That is, $\mathscr{Z}=\mathscr{Z}^{*}$. $\square$

Example 2. Let $\mho=[0, \infty)$ be equipped with the metric $\varphi($ $\mathfrak{R}, \mathcal{Y})=|\mathfrak{R}-\mathcal{Y}|$ for all $\mathfrak{R}, \mathcal{Y} \in \mathcal{J}$. Suppose $\mathfrak{R} \perp \mathcal{Y}$ if $\mathfrak{R} \mathscr{Y}$ $=\mathfrak{R}$. Let $\mathfrak{G}: \mho \times \mho \longrightarrow \mho$ be defined by

$$
\mathfrak{G}(\mathfrak{R})= \begin{cases}\frac{\mathfrak{R}}{\mathfrak{R}+1} & \text { if } \mathfrak{R} \in[0,1] \\ 2 \mathfrak{R} & \text { if } \mathfrak{R} \in(1, \infty)\end{cases}
$$

Define $\alpha: \mathfrak{R} \times \mathfrak{R} \longrightarrow[0, \infty)$ and $\hbar:[0, \infty) \longrightarrow[0,1]$ by

$$
\begin{gathered}
\alpha(\mathfrak{R}, \mathscr{Y})= \begin{cases}1 & \text { if } \mathfrak{R}, \mathscr{Y} \in[0,1] \\
0 & \text { otherwise }\end{cases} \\
\hbar(\vartheta)=\frac{1}{1+\vartheta} .
\end{gathered}
$$

Clearly, $(\mho, \varphi)$ is an $O$-complete metric space and $\perp$ -preserving. We claim that $\mathfrak{G}$ is an orthogonal triangular $\alpha$ -admissible mapping. Let $\mathfrak{R}, \mathcal{Y} \in \mho$. If $\mathfrak{R} \perp \mathcal{Y}, \alpha(\mathfrak{R}, \mathcal{Y}) \geq 1$, then $\mathfrak{R}, \mathscr{Y} \in[0,1]$. Also, for $\mathfrak{R} \in[0,1]$, we have $\mathfrak{G} \mathfrak{R} \leq 1$. It yields that $\alpha(\mathfrak{G} \mathfrak{R}, \mathfrak{G} \mathscr{Y}) \geq 1$.

Let $\mathfrak{R}, \mathscr{Y}, \mathscr{Z} \in \mho$. If $\mathfrak{R} \perp \mathscr{Z}, \alpha(\mathfrak{R}, \mathscr{Z}) \geq 1$ and $\mathscr{Z} \perp \mathscr{Y}, \alpha($ $\mathscr{Z}, \mathscr{Y}) \geq 1$, then, $\mathfrak{R}, \mathscr{Y}, \mathscr{Z} \in[0,1]$. Also, for $\mathfrak{R} \in[0,1]$, we get $\mathfrak{G} \mathfrak{R} \leq 1$. Thus, $\mathfrak{R} \perp \mathcal{Y}, \alpha(\mathfrak{G} \mathfrak{R}, \mathfrak{G} \mathscr{Y}) \geq 1$. Hence, the statement is satisfied. Due to the above, $\alpha(0, \mathfrak{G} 0) \geq 1$.

Now, let $\left\{\Re_{\mathfrak{n}}\right\}$ be a sequence in $\mho$ so that $\alpha\left(\Re_{\mathfrak{n}}, \mathfrak{R}_{\mathfrak{n}+1}\right.$ ) $\geq 1$ for all $\mathfrak{n} \geq 0$ and $\mathfrak{R}_{\mathfrak{n}} \longrightarrow \mathfrak{R}$ as $\mathfrak{n} \longrightarrow \infty$, then, $\left\{\mathfrak{R}_{\mathfrak{n}}\right\}$ $\subset[0,1]$, hence, $\mathfrak{R} \in[0,1]$. This implies that $\alpha(\mathfrak{R}, \mathfrak{G} \mathfrak{R}) \geq 1$.
Let $\mathfrak{R}, \mathcal{Y} \in[0,1]$ and $\mathscr{Y} \geq \mathfrak{R}$. We get

$$
\begin{aligned}
& (\varphi(\mathfrak{G R}, \mathfrak{G} \mathscr{Y})+\mathfrak{l})^{\alpha(\mathfrak{R}, \mathfrak{G}) \alpha(\mathscr{Y}, \mathfrak{G} \mathscr{Y})}=\mathfrak{G} \mathcal{Y}-\mathfrak{G} \mathfrak{R}=\frac{\mathscr{Y}}{\mathscr{Y}+1} \\
& -\frac{\mathfrak{R}}{\mathfrak{R}+1}+\mathfrak{l}=\frac{\mathscr{Y}-\mathfrak{R}}{(1+\mathfrak{R})(1+\mathscr{Y})}+\mathfrak{l} \leq \frac{\mathscr{Y}-\mathfrak{R}}{1+\mathscr{Y}-\mathfrak{R}} \\
& \quad+\mathfrak{l}=\hbar(\varphi(\mathfrak{R}, \mathscr{Y})) \varphi(\mathfrak{R}, \mathscr{Y})+\mathfrak{l} .
\end{aligned}
$$

$$
\begin{gathered}
\text { Otherwise, } \alpha(\mathfrak{R}, \mathfrak{G} \mathfrak{R}) \alpha(\mathcal{Y}, \mathfrak{G} \mathscr{Y})=0 \text { and so } \\
(\varphi(\mathfrak{G R}, \mathfrak{G} \mathscr{Y})+\mathfrak{l})^{\alpha(\mathfrak{R}, \mathfrak{G} \mathfrak{R}) \alpha(\mathscr{Y}, \mathfrak{G} \mathscr{Y})}=1 \leq \hbar(\varphi(\mathfrak{R}, \mathscr{Y})) \varphi(\mathfrak{R}, \mathcal{Y})+\mathfrak{l} .
\end{gathered}
$$

Hence, all the hypotheses of Theorem 7 are satisfied, and $\mathfrak{G}$ has a unique fixed point, $\mathfrak{R}=0$.

\section{Application}

Let $\mho=C\left[\lambda_{1}, \lambda_{2}\right]$ be a set of all real continuous functions on $\left[\lambda_{1}, \lambda_{2}\right]$ equipped with metric $\varphi(\mathfrak{R}, \mathscr{Y})=|\mathfrak{R}-\mathscr{Y}|$ for all $\mathfrak{R}$ , $\mathscr{Y} \in C\left[\lambda_{1}, \lambda_{2}\right]$. Then, $(\mho, \varphi)$ is a complete metric space. Consider the orthogonality relation $\perp$ on $\mho$ given as

$\mathfrak{R} \perp \mathscr{Y} \Leftarrow \mathfrak{R}(\Omega) \mathscr{Y}(\Omega) \geq \mathfrak{R}(\Omega)$ or $\Re(\Omega) \mathscr{Y}(\Omega) \geq \mathscr{Y}(\Omega), \forall \Omega \in\left[\lambda_{1}, \lambda_{2}\right]$. tion

Now, we consider the nonlinear Fredholm integral equa-

$$
\mathfrak{R}(\Omega)=\mathfrak{v}(\Omega)+\frac{1}{\lambda_{2}-\lambda_{1}} \int_{\lambda_{1}}^{\lambda_{2}} j(\Omega, \mathfrak{G}, \mathfrak{R}(\mathfrak{S})) d s,
$$

where $\Omega, \mathfrak{G} \in\left[\lambda_{1}, \lambda_{2}\right]$. Assume that $j:\left[\lambda_{1}, \lambda_{2}\right] \times\left[\lambda_{1}, \lambda_{2}\right] \times \mathfrak{R}$ $\longrightarrow \mathbb{R}$ and $\mathfrak{v}:\left[\lambda_{1}, \lambda_{2}\right] \longrightarrow \mathbb{R}$ continuous, where $\mathfrak{b}(\Omega)$ is a given function in $\mho$.

Theorem 11. Suppose that $(\mho, d)$ is an O-complete metric space equipped with the metric $\varphi(\mathfrak{R}, \mathcal{Y})=|\mathfrak{R}-\mathcal{Y}|$ for all $\mathfrak{R}, \mathcal{Y} \in \mho$ and $\mathfrak{W}: \mho \longrightarrow \mho$ is an orthogonal continuous operator on $\mho$ defined by

$$
\mathfrak{G} \Re(\Omega)=\mathfrak{v}(\Omega)+\frac{1}{\lambda_{2}-\lambda_{1}} \int_{\lambda_{1}}^{\lambda_{2}} j(\Omega, \mathfrak{g}, \mathfrak{R}(\mathfrak{g})) d s,
$$

for all $\mathfrak{R}, \mathcal{Y} \in \mho$ with $\mathfrak{R} \neq \mathcal{Y}$ and $\mathfrak{G}, \Omega \in\left[\lambda_{1}, \lambda_{2}\right]$ satisfying the following inequality

$$
|j(\Omega, \mathfrak{G}, \mathfrak{G} \mathfrak{R}(\mathfrak{g}))-j(\Omega, \mathfrak{G}, \mathfrak{G} \mathscr{Y}(\mathfrak{s}))| \leq \frac{|\mathfrak{R}-\mathscr{Y}|}{1+|\mathfrak{R}-\mathcal{Y}|},
$$

then, the integral operator defined by (78) has a unique solution. 
Proof. We define $\alpha: \mathfrak{R} \times \mathfrak{R} \longrightarrow[0, \infty)$ such that $\alpha(\mathfrak{R}, \mathscr{Y})$ $=1$ for all $\mathfrak{R}, \mathcal{Y} \in \mho$ and $\hbar:[0, \infty) \longrightarrow[0,1]$ defined by

$$
\hbar(t)=\frac{1}{1+t}
$$

Therefore, $\mathfrak{G}$ is orthogonally triangular $\alpha$-admissible. Now, we show that $\mathfrak{G}$ is $\perp$-preserving. For each, $\mathfrak{R}, \mathcal{Y} \in \mathcal{\mho}$ with $\mathfrak{R} \perp \mathcal{Y}$ and $\Omega \in[a, b]$, we have

$$
\mathfrak{G} \mathfrak{R}(\Omega)=\mathfrak{v}(\Omega)+\frac{1}{\lambda_{2}-\lambda_{1}} \int_{\lambda_{1}}^{\lambda \lambda_{2}} j(\Omega, \mathfrak{G}, \mathfrak{R}(\mathfrak{g})) d s \geq 1
$$

Accordingly, $[(\mathfrak{G} \Re)(\Omega)][(\mathfrak{G} \mathscr{Y})(\Omega)] \geq(\mathfrak{G} \mathcal{Y})(\Omega)$ and so $(\mathfrak{G} \mathcal{Y})(\Omega) \perp(\mathfrak{G} \mathcal{Y})(\Omega)$. Then, $\mathfrak{G}$ is $\perp$-preserving. Clearly, $\mathfrak{G}$ is orthogonally continuous. Let $\mathfrak{R}, \mathscr{Y} \in \mho, \mathfrak{l} \geq 1$ with $\mathfrak{R} \perp \mathcal{Y}$ . Suppose that $\mathfrak{G}(\mathfrak{R}) \neq \mathfrak{G}(\mathscr{Y})$. Using (78), we derive

$$
\begin{aligned}
& (\varphi(\mathfrak{G} \mathfrak{R}, \mathfrak{G} \mathscr{Y})+\mathfrak{l})^{\alpha(\mathfrak{R}, \mathfrak{G} \mathfrak{R}) \alpha(\mathscr{Y}, \mathfrak{G} \mathscr{Y})}=\varphi(\mathfrak{W} \mathfrak{R}, \mathfrak{W} \mathscr{Y}) \\
& +\mathfrak{l}=|\mathfrak{G} \mathfrak{R}-\mathfrak{G} \mathscr{Y}|+\mathfrak{l}=\frac{1}{\left|\lambda_{2}-\lambda_{1}\right|} \mid \int_{\lambda_{1}}^{\lambda_{2}} j(\Omega, \mathfrak{G}, \mathfrak{G} \mathfrak{R}(\mathfrak{g})) d \mathfrak{s} \\
& -\int_{\lambda_{1}}^{\lambda_{2}} j(\Omega, \mathfrak{G}, \mathfrak{G} \mathscr{Y}(\mathfrak{g})) d s\left|+\mathfrak{l} \leq \frac{1}{\left|\lambda_{2}-\lambda \lambda_{1}\right|} \int_{\lambda_{1}}^{\lambda_{2}}\right| j(\Omega, \mathfrak{g}, \mathfrak{G} \mathfrak{R}(\mathfrak{g})) \\
& -j(\Omega, \mathfrak{g}, \mathfrak{G} \mathscr{Y}(\mathfrak{g})) \mid d s+\mathfrak{l} \leq \frac{1}{\left|\lambda_{2}-\lambda_{1}\right|} \int_{\lambda_{1}}^{\lambda_{2}} \frac{|\mathfrak{R}-\mathscr{Y}|}{1+|\mathfrak{R}-\mathscr{Y}|} d s+\mathfrak{l}, \\
& =\frac{|\mathfrak{R}-\mathscr{Y}|}{1+|\mathfrak{R}-\mathscr{Y}|}+\mathfrak{l}=\hbar(\varphi(\Re, \mathscr{Y})) \varphi(\mathfrak{R}, \mathscr{Y})+\mathfrak{l} .
\end{aligned}
$$

Hence, all the conditions of Theorem 7 are satisfied, and so the integral operator $\mathfrak{G}$ defined by (78) has a unique solution. $\square$

\section{Conclusion}

The idea of $\alpha$-admissibility on $O$-complete metric spaces was introduced in this article, and some fixed point theorems were demonstrated. An illustrative example is provided that shows the validity of the hypotheses and the degree of usefulness of our findings.

\section{Data Availability}

No data were used to support this study.

\section{Conflicts of Interest}

The authors declare that there is not any competing interest regarding the publication of this manuscript.

\section{Authors' Contributions}

All authors contributed equally to the writing of this paper. All authors read and approved the final manuscript.

\section{References}

[1] D. Wardowski, "Fixed points of a new type of contractive mappings in complete metric spaces," Fixed point theory and applications, vol. 2012, no. 1, Article ID 206, 2012.

[2] M. E. Gordji, M. Ramezani, M. De La Sen, and Y. J. Cho, “On orthogonal sets and Banach fixed point theorem," Fixed Point Theory, vol. 18, no. 2, pp. 569-578, 2017.

[3] M. Eshaghi Gordji and H. Habibi, "Fixed point theory in generalized orthogonal metric space," Journal of Linear and Topological Algebra (JLTA), vol. 6, no. 3, pp. 251-260, 2017.

[4] K. Sawangsup, W. Sintunavarat, and Y. J. Cho, "Fixed point theorems for orthogonal F-contraction mappings on O-complete metric spaces," Journal of Fixed Point Theory and Applications, vol. 22, no. 1, p. 10, 2020.

[5] M. Eshaghi and H. Habibi, "Fixed point theory in $\varepsilon$-connected orthogonal metric space," Shand Communications in Mathematical Analysis (SCMA), vol. 16, no. 1, pp. 35-46, 2019.

[6] N. B. Gungor and D. Turkoglu, "Fixed point theorems on orthogonal metric spaces via altering distance functions," AIP Conference Proceedings, vol. 2183, article 040011, 2019.

[7] O. Yamaod and W. Sintunavarat, "On new orthogonal contractions in b-metric spaces," international journal of pure mathematics, vol. 5, 2018.

[8] K. Javed, H. Aydi, F. Uddin, and M. Arshad, "On orthogonal partial -metric spaces with an application," Journal of Mathematics, vol. 2021, Article ID 6692063, 7 pages, 2021.

[9] K. Sawangsup and W. Sintunavarat, "Fixed point results for orthogonal Z-contraction mappings in O-complete metric Spaces," International Journal of Applied Physics and Mathematics, vol. 10, no. 1, pp. 33-40, 2020.

[10] T. Senapati, L. K. Dey, B. Damjanović, and A. Chanda, "New fixed results in orthogonal metric spaces with an application, Kragujevac," Journal of Mathematics, vol. 42, no. 4, 516 pages, 2018.

[11] M. Gunaseelan, G. Arul Joseph, L. N. Mishra, and V. N. Mishra, "Fixed point theorem for orthogonal F-suzuki contraction mapping on a $\mathrm{O}$-complete metric space with an application," Malaya Journal of Matematik, vol. 1, pp. 369-377, 2021.

[12] I. Beg, G. Mani, and A. J. Gnanaprakasam, "Fixed point of orthogonal F-Suzuki contraction mapping on O-complete bmetric spaces with applications," Journal of Function spaces, vol. 2021, Article ID 6692112, 12 pages, 2021.

[13] F. Uddin, K. Javed, H. Aydi, U. Ishtiaq, and M. Arshad, "Control fuzzy metric spaces via orthogonality with an application," Journal of Mathematics, vol. 2021, Article ID 5551833, 12 pages, 2021.

[14] M. U. Ali, H. Aydi, and M. Alansari, "New generalizations of set valued interpolative Hardy-Rogers type contractions in bmetric spaces," Journal of Function Spaces, vol. 2021, Article ID 6641342, 8 pages, 2021.

[15] N. Hussain, E. Karapinar, P. Salimi, and F. Akbar, " $\alpha$-Admissible mappings and related fixed point theorems," Journal of Inequalities and Applications, vol. 2013, no. 1, 113 pages, 2013.

[16] M. Ramezani, "Orthogonal metric space and convex contractions," International Journal of Nonlinear Analysis and Applications, vol. 6, no. 2, pp. 127-132, 2015. 